\title{
Smart e-commerce integration with recommender systems
}

\author{
Yin Zhang ${ }^{1} \cdot{\text { Haider } \text { Abbas }^{2} \text { (1) } \cdot \text { Yi Sun }}^{3}$ \\ Received: 4 April 2019 / Accepted: 5 April 2019 / Published online: 17 May 2019 \\ (C) Institute of Applied Informatics at University of Leipzig 2019
}

With the advancement of information technology, ecommerce is developing rapidly and has also paved the ways for seamless accessibility of e-commerce related processes anytime and anywhere. Assisted by cloud computing, the Quality of Service (QoS) and Quality of Experience (QoE) of e-commerce have experienced significant improvements. Supported by big data, e-commerce is becoming smarter that more intelligent services and applications are emerging. Especially, the role of recommender systems is vital in terms of implementing personalized and intelligent services and has great significance in the development of smart e-commerce. The core of the recommender system is information filtering and information retrieval to extract item features and model users' interest for recommending suitable contents to user. Such system aims at minimizing search costs of customers and easing the discovery of preferred products based on explicit and implicit feedback given by consumers. Recommender systems actually makes use of consumer's feedback and buying behavior information in

This article is part of the Topical Collection on Smart e-Commerce Integration with Recommender Systems.

Responsible Editor: Rainer Alt

Yin Zhang

yin.zhang.cn@ieee.org

Haider Abbas

dr.h.abbas@ieee.org

Yi Sun

sybupt@bupt.edu.cn

1 Zhongnan University of Economics and Law, Wuhan, Hubei Sheng, China

2 National University of Sciences and Technology, Islamabad, Pakistan

3 Beijing University of Posts and Communications, Beijing, China order to infer preferences of customers and recommend products automatically. These systems generally work on collaborative and content-based filtering mechanisms. Majority of the recommender systems employ agents to elicit user's interests and preferences based on past transactional data from social media profile.

Although this innovative process of recommender systems exhibits high efficiency, there are several great challenges including data sparsity, predictable recommendations, cold start problem, incorporation of content, over specialization problem, hybrid data and scalability to enable humanized services for complex commerce environments and various user demands. Due to unavailability of optimal information, it becomes difficult for recommender systems to extract item features and model interest of users for suggesting suitable contents to user. Therefore, it is necessary to develop an efficient, objective and accurate recommender system to provide a strong foundation for smart e-commerce.

This special issue features five selected papers with high quality. The first article, "Smart e-commerce systems: current status and research challenges", authored by Zhiting Song, Yanming Sun, Jiafu Wan, and Lingli Huang describes a holistic architecture of these systems and analyzes the main enablers underlying the development of smart e-commerce systems in terms of internet of things (IoT), social media, mobile internet, big data analytics and cloud computing (Song et al. 2019). Moreover, the key challenges and issues pertaining to current smart e-commerce systems were presented, while some possible research directions were also proposed.

The second article titled "PIRASA: Strategic Protocol Selection for E-Commerce Agents" presents an agentbased simulation environment for studying how autonomous agents can best interact with each other to exchange goods in e-commerce marketplaces (Hopkins et al. 2019). PIRASA allows agents to determine which protocol is more beneficial via experimentation in different settings, and supports the customization of agent attributes, which govern the agent's behavior. 
In the next article with the title "Effects of Sentiment on Recommendations in Social Network", the authors adopted a sentiment word database to extract sentiment-related data from microblog posts for investigating the effect of different types of sentiment-related words on product recommendations (Hsu et al. 2019). The results could be expected to assist product or service marketers who use Plurk or similar microblogging platforms better focus their limited financial resources on potential online customers to achieve maximum sale revenue.

To improve online shopping experience for consumers, increase sales for merchants and achieve effective warehousing and delivery, the fourth article titled "User Behavior Modeling, Recommendations, and Purchase Prediction During Online Shopping Festivals" investigates user online browsing and purchasing behaviors, and predicts purchasing actions during a large shopping festival in China (Zeng et al. 2019). Based on the obtained user behaviors and massive data, it applied collaborative filtering based method to recommend items for different consumers, and predicted whether purchase would happen.

The last article titled "Fractional Stochastic Gradient Descent for Recommender Systems" proposes a fractional variant of the standard stochastic gradient descent to make recommendations more precise and efficient. Through experimental results, it shows that the proposed method performed significantly well in terms of estimated accuracy and convergence as compared to the standard approaches (Khan et al. 2019).
Acknowledgements The guest editors are thankful to all the authors who submitted their research work to this special issue. We also appreciate the contribution of many experts in the field who have participated in the review process and provided constructive suggestions to the authors to improve the contents and presentations of the articles. In particular, we would like to thank Professor Rainer Alt and Hans-Dieter Zimmermann, the Editors-in-Chief, and staff members for their support and helpful suggestions during all stages of concluding this special issue.

\section{References}

Hopkins, J., Kafali, Ö., Alrayes, B., \& Stathis, K. (2019). Pirasa: strategic protocol selection for e-commerce agents. Electronic Markets, 29(2). https://doi.org/10.1007/s12525-018-0307-4 .

Hsu, P.-Y., Lei, H.-T., Huang, S.-H., Liao, T. H., Lo, Y.-C., \& Lo, C.-C. (2019). Effects of sentiment on recommendations in social network. Electronic Markets, 29(2). https://doi.org/10.1007/s12525-018-0314-5 .

Khan, Z. A., Chaudhary, N. I., \& Zubair, S. (2019). Fractional stochastic gradient descent for recommender systems. Electronic Markets, 29(2). https://doi.org/10.1007/s12525-018-0297-2 .

Song, Z., Sun, Y., Wan, J., Huang, L., \& Zhu, J. (2019). Smart e-commerce systems: current status and research challenges. Electronic Markets, 29(2). https://doi.org/10.1007/s12525-017-0272-3 .

Zeng, M., Cao, H., Chen, M., \& Li, Y. (2019). User behaviour modeling, recommendations, and purchase prediction during shopping festivals. Electronic Markets, 29(2). https://doi.org/10.1007/s12525018-0311-8 .

Publisher's note Springer Nature remains neutral with regard to jurisdictional claims in published maps and institutional affiliations. 